\title{
BIOPOLÍTICA Y SEGURIDAD \\ NACIONAL EN LA WEB 2.0. BREVE ANÁLISIS DE UN CASO ECUATORIANO
}

BIOPOLITICS AND NATIONAL SECURITY IN WEB 2.0: A BRIEF ANALYSIS OF AN ECUADORIAN CASE STUDY

\section{FREDY RIVERA VÉLEZ GILDA ALICIA GUERRERO SALGADO**}

Recibido 10 de junio del 2016 Aceptado 10 de agosto del 2016

\footnotetext{
* Flacso Ecuador, Quito, Ecuador (frivera@flacso.edu.ec)

** Pontifica Universidad Católica del Ecuado, Quito. Ecuador (gguerrero185@puce.edu.ec)
} 



\title{
BIOPOLÍTICA Y SEGURIDAD NACIONAL EN LA WEB 2.0. BREVE ANÁLISIS DE UN CASO ECUATORIANO
}

\author{
BIOPOLITICS AND NATIONAL SECURITY \\ IN WEB 2.0: A BRIEF ANALYSIS OF AN ECUADORIAN \\ CASE STUDY
}

FREDY RIVERA VÉLEZ, GILDA ALICIA GUERRERO SALGADO

\begin{abstract}
PALABRAS CLAVE: Biopolítica, seguridad nacional, redes sociales, biopoder,
\end{abstract} ciberseguridad, acción colectiva.

\begin{abstract}
KEY WORDS: Biopolitics, National Security, social networks, biopower, cybersecurity, collective action
\end{abstract}

\section{RESUMEN}

Internet es en un espacio político y de acción colectiva que influye y legitima las discusiones ciudadanas en lo público offline. Estas características se consideran ocasionalmente amenazas para el Estado que interviene, legisla, monitorea o bloquea la comunicación para "devolver la salud" al espacio virtual como una muestra de biopoder que se aplica a la seguridad nacional. Con el fin de ilustrar este fenómeno desde América Latina, el presente texto ana- liza el caso del registro de marca sobre frases relativas al motín policial de 2010 en Ecuador. A través de este ejemplo, se pretende señalar cómo esta acción legal, que incluyó una expresión inicialmente propuesta en Twitter (hashtag), es una reacción segurizadora que tiene un fundamento biopolítico que se ampara en el principio de retirar un elemento dañino para mantener la integridad, el poder y la capacidad de control del Estado. 


\begin{abstract}
Internet has become a new space for political and collective action. Thus, it influences and legitimizes citizens' discussions that take place within the offline sphere. These features are occasionally considered threats to the State which, under perceived danger, legislates, monitors or blocks communications to "restore health" to virtual space as a sign of biopower applied to national security. In order to illustrate this phenomenon from a Latin American

of the trademark registration of phrases related to the police mutiny that took place in Ecuador in 2010. Through this example, it is intended to point out how this legal action, which included an expression initially proposed on Twitter (a hashtag), is a securitizing reaction of the State with a biopolitical basis, sustained by the principle of removing a harmful element from the political ecosystem to maintain the integrity, power, and controllability of the State.
\end{abstract} perspective, this paper analyzes the case

\title{
INTRODUCCIÓN Y METODOLOGÎA
}

\section{Internet y seguridad nacional, una relación compleja}

Internet ya no es una red "cartelera"; una red sencilla de exposición de datos, pues se complejiza para transformarse en varias redes intrincadas $y$ superpuestas, en las que los usuarios toman una identidad, una calidad de "cibersujeto" político (Morueco, s.f.). Así, son capaces de reflexión y acciones, de manera que vuelven al espacio público offline y al espacio virtual online, en un continuo con posibilidades de influenciar o al menos delinear, ciertas realidades sociales (Levy, 2011).

De esta forma, Internet daría paso a patrones de red y acción colectiva en los que:

Se desarrolla una estructura electrónica reticular que vincula diferentes computadoras y a partir de allí diversos sujetos y actores sociales (individuales, organizacionales) entre sí, donde cada uno adquiere la posición de nodo y las relaciones que los vinculan pueden ser asumidas como arcos o aristas. (Román y Urán, 2007:6)

De esta forma, lo que se conoce como Internet se transformó en esta red 
ReVista PUCE, ISSN 1390-7719. Núm.103. 3 de mAYO de 2016-

3 de noviembre de 2016. Fredy Rivera Vélez, Gilda Alicia

mundial, la Red 2.0' y es ahora un instrumento para la acción colectiva que agrupa a diferentes entidades e individuos que buscan una herramienta de participación. Si bien la tecnología no es una base de las fuerzas sociales, es importante señalar que la primera es producto paralelo de las últimas, de manera que los procesos políticos e incluso históricos, pueden ser comprendidos a través de estos nuevos recursos (Castells, 2002). Por lo tanto, diversos fenómenos sociales actuales tienen un punto de anclaje tecnológico: lo sociopolítico no puede estar al margen de la tendencia, como muestra la cada vez mayor participación de las mencionadas redes en el quehacer de la comunicación política.

La importancia de esta Web 2.0 se puede observar en todo el sistema internacional. Varios Estados tienen al menos una página web de Presidencia e incluso utilizan a las redes sociales para su acción gubernamental. Un ejemplo lo presenta Savanoni (2010), cuando relata cómo el Presidente de Estados Unidos, Barack Obama, abrió la red de la Casa

' El concepto de Web 2.0 está en línea a la dinámica de Internet como una plataforma de software en continuo desarrollo. Tiene una arquitectura de participación y cocreación. Los datos son dinámicos y son susceptibles a la mezcla y a la transformación. Debido al número de dispositivos y a la inteligencia colectiva, la Red 2.0 crece y se desarrolla de manera rentable, lo cual apoya a la difusión de toda clase de actividades cotidianas, incluida la política. (O’Reilly, 2006).
Blanca. La estrategia tuvo dos fases: en la primera se creó un portal del gobierno a través de Creative Commons, licencias que permiten el uso de contenidos para los ciudadanos. La otra fase, en cambio, quedó en una iniciativa que duró desde 2008 a 2010: White House2 (http://www. whitehouse2.org/), un proyecto de red social ideado por Jim Gilliam en el cual:

...cualquier estadounidense pudiera hacer una lista de los retos a que se enfrentaba el país y exponer su opinión sobre cuáles eran sus máximas prioridades. La intención de Gilliam era constituir una gobernanza virtual que ofreciera al presidente Obama un valioso instrumento de consulta popular. El sitio web se lanzó, pero no se incorporó al conjunto de estrategias de comunicación del Presidente. Esta iniciativa sigue en marcha y hoy en día es un entorno en el cual diez mil estadounidenses debaten sobre cuáles deben ser las prioridades del Gobierno actual- (Savanoni, 2010: 23).

Es decir, Internet se volvió un instrumento político, un elemento para lograr participación ciudadana y para apoyar el trabajo de los gobiernos. No obstante, en el otro lado del espectro, desde hace menos de una década, esta 
tendencia comenzó a desarrollarse hacia otras instancias de la política que están en un bando diferente: la seguridad nacional.

La importancia de esta acción colectiva en la Web 2.0 se puede observar con el advenimiento de las llamadas Revoluciones 2.0, es decir, las manifestaciones "movilizadoras de la respuesta ciudadana" (Navarro y García, 2011), que desde 2011 crecen y se multiplican gracias a la inmediatez, rapidez y carácter masivo de plataformas como Twittery Facebook. Internet dio forma a la verdadera red (entendido el término bajo los conceptos detrás de las palabras inglesas Web o Net); es decir, formó una sociedad reticular que va a recibir impulsos externos mientras que es capaz de crear nuevos significados y sentidos. Así, se forma una esfera que influye en realidades, pensamientos y reacciones de amplio alcance (Castells, 2002; Maldonado y Urán, 2007).

Por lo tanto, estos espacios colectivos se orientan a uno o varios intereses comunes. Se sistematizan y catalizan organización, movilización, oportunidades, conductividad, tensión, poder e identidad gracias a diversos actores y agentes que trabajan en cooperación (e incluso en conflicto), por una razón, con un cómo específico en la búsqueda de beneficios (Beriain, 1996, citado por Maldonado y Urán, 2007). La tendencia es fácil de ligar a las ya mencionadas grandes movilizaciones: desde el Magreb, con la llamada Primavera Árabe, hasta Madrid, con el movimiento de los Indignados las redes sociales plantan resistencia en el ciberespacio y fuera de él, a la crisis y a visiones gubernamentales².

Ahora, si bien la conectividad e inteligencia colectiva de la Red $2.0 \mathrm{im}$ plican un uso político de Internet desde los ciudadanos -y apoyan a la comunicación política desde los gobiernos- estos fenómenos también se transforman en un problema de seguridad estatal. El Estado ya no puede manejar solamente el espacio público del debate tradicional, sino que también debe enfrentarse a los fenómenos del espacio virtual, que se vincula a la sociedad de información y al movimiento de recursos a gran escala (Habermas, 1981; Castells, 2005; 2010. Citados por Puyosa, 2012).

Frente al dilema, el Estado tiene como primera reacción el blindaje de sí mismo, de su espacio y de su integridad en todo sentido. Es lo que Buzan, Wæver \& De

2 Existen más de 1500 millones de usuarios en Facebook (Moreno, 2016). Sorprende el crecimiento de las redes en países de poco acceso. China, por ejemplo, tiene importantes controles, pero también prolíficos ciberactivistas. En este contexto aparecen las "revoluciones 2.0": acciones colectivas que terminaron gobiernos y que comenzaron en países como Túnez y Egipto, todavía en desarrollo. (Sedra, 2011; ver Watts, 2006). 
ReVista PUCE, ISSN 1390-7719. Núm.103. 3 de mAYO de 2016-

3 de noviembre de 2016. Fredy Rivera Vélez, Gilda Alicia

GUERRERO SALGADO. PP. 233-252

Wilde (1998) llamaron segurización $n^{3}$ : la protección en caso de cualquier movimiento que podría vulnerar de forma directa al Estado, más allá de los marcos conocidos.

Es decir, esa segurización se da en el señalamiento y acción política sobre un hecho, que queda en la agenda estatal. El Estado utiliza el discurso para aumentar la incidencia de un proceso y mostrar a la ciudadanía su posible peligro para los intereses nacionales (Rivera, 2012). Como un médico y en un ejercicio de biopolítica, el Estado señala y retira lo que puede perjudicar a su organismo para mantener su identidad, su vida. Se bloquea y se encierra a la subjetividad humana para disciplinar a los individuos con el objetivo de lograr un orden del cuerpo estatal (Foucault, 2006).

En este contexto, el presente artículo se pregunta si es posible encontrar

\footnotetext{
${ }^{3}$ Se justifica el neologismo de la "segurización" como la traducción más acertada del concepto securitization. Otra opción utilizada en la Academia es la de "seguritización": "Entenderemos por seguritización, la acción de relacionar un tema directamente con el ámbito de la seguridad, para que, de esta forma, el tema adquiera prioridad en los procesos de toma de decisiones políticos gubernamentales (...). El término inglés "securitization" cuya autoría le corresponde a Oleg Waever, no es fácil de traducir al español, se encuentra traducido como "seguritización" en un artículo de Andrew Hurrel, "Seguridad y Violencia en América Latina: Un análisis conceptual". Foro Internacional. Vol. XXXVIII. № 1. Enero-marzo 1998. Además, en el artículo de Esther Barbé y Orieta Perni, "Más Allá de la Seguridad Nacional", en Carlos de Cueto y Javier Jordán, Introducción a los Estudios de Seguridad y Defensa, Editorial Comares 2001; el término "securitization" se encuentra traducido como segurización. En este caso la opción seguritizar nos parece la más adecuada." (Griffiths, 2007).
}

casos de segurización de la Web 2.0 en Ecuador, por lo que afirma como hipótesis que las redes sociales en Internet, observadas por el Estado como parte de su integridad, se encuentran en un incipiente proceso de regularización, mediación y hasta de segurización; no tanto por su capacidad de crear o modificar la realidad, sino por su potencialidad a mediano y largo plazo de atentar contra la "buena salud" del Estado Nacional.

Para probar esta afirmación en el contexto deseado se toma el caso del registro de marca sobre la "hashtag \#30S"4 por parte del actual gobierno ecuatoriano. Este acrónimo, etiqueta de Twitter, aparece en línea para organizar en la red social los hechos de la rebelión policial del 30 de septiembre de 2010 en Ecuador (desde el tuitero @earcos). El Estado ecuatoriano, como se explicará en el siguiente apartado, aisló este elemento para mantener su integridad y su salud, a través de la apropiación del símbolo.

La investigación, que parte de un estudio más amplio, ${ }^{5}$ se realizó a través del análisis del discurso mediático. Para

${ }^{4}$ La hashtag \#30S se utiliza en este texto solamente con fines de divulgación académica, fuera del registro mencionado en este artículo (30SMR).

${ }^{5}$ Este artículo se inscribe en un estudio, inédito, sobre la relación entre la seguridad, los movimientos sociales, y el uso de la Web 2.0 y se enmarca como un resultado alterno dentro del trabajo doctoral de Gilda Guerrero, dirigida por Fredy Rivera Vélez (proceso doctoral en UNCUYO, Argentina). Un artículo de divulgación general sobre otro eje temático paralelo, contiene algunos autores y datos señalados en el presente documento (Rivera, F. \& Guerrero, G., 2014). 
esto, se toma una muestra de las declaraciones de prensa realizadas por los implicados en el registro de la "hashtag \#30S" y sus analistas. Con esta metodología se observaron las expresiones que señalan: 1) un deseo de construcción de sentido de la realidad que se refiere a la protección del Estado con el registro de marca y 2) las microestructuras textuales, elementos y significantes que indican la potencialidad del gobierno para realizar la acción legal y el deseo de "mantener sano" al país a través de la separación del acrónimo. Se creó este sistema con el análisis del discurso de Van Dijk (1997) y la experiencia de Sánchez (2011).

\section{PRESENTACIÓN DE CASO}

Con este método y para poner a prueba la hipótesis, se toma el caso del uso de la red social Twitter en Ecuador durante el señalado 30 de septiembre de 2010, día en que el país vivió una jornada de incertidumbre, cuando un grupo de policías se amotinaron al perder privilegios económicos como parte de las medidas del gobierno del presidente ecuatoriano Rafael Correa. (De la Torre, 2011).

Los amotinados intentaban evitar el cambio de su sistema de pagos y recompensas. La reforma se inscribió dentro del Sumak Kawsay o "Buen Vivir", contexto en el que se reformó la Constitución ecuatoriana: "La Carta Magna aprobada en el 2008 afirmó la soberanía nacional y popular, fortaleció al Estado, realizó reformas en el régimen político e introdujo las orientaciones de un nuevo modelo de desarrollo bajo la noción del "sumak kawsay", dirigida a superar la larga noche neoliberal"' (Ortiz, 2011: 26).
En la jornada, según informa la web oficial de hecho, "Los policías amotinados bombardean con gases lacrimógenos las inmediaciones del Hospital de la Policía impidiendo la salida del Mandatario"s. Paralelamente, mientras que en cadena nacional de la televisión pública (ECTV) se relataban los hechos, las redes sociales funcionaron sin restricción y se volvieron una vía alternativa de comunicación (Coronel, 2011).

El acto insurrecto conmocionó al país y desató sanciones disciplinarias y judiciales que continúan en indagación?

${ }^{6}$ Véase: http://coordinacion.secom.gob.ec/decretos/el30s/index.html\#, donde se observa la cronología de los hechos, perteneciente al archivo: http://www.el30s.com/, apartado Secuestro en Hospital.

${ }^{7}$ Mientras se escribe el presente artículo, todavía se realizan indagaciones de los implicados (Crónica, 2016, 18 de mayo). Cabe anotar que este trabajo no se enmarca en la discusión de los hechos de la revuelta, sino en información meramente académica alrededor del registro de marca. 
ReVista PUCE, ISSN 1390-7719. Núm.103. 3 de mAYO de 2016-

3 de noviembre de 2016. Fredy Rivera Vélez, Gilda Alicia

Paralelamente, la línea comunicacional de las redes en Internet, en especial Twitter $^{8}$, se subrayó después del 30 de septiembre de 2010. Ese día, los acontecimientos se construyeron y se reflejaron en esta red social. Durante la jornada de la revuelta, Twitter se marcó con la hashtag \#3059, mediante propuesta de un comunicador ecuatoriano (Gráfico 1). Como toda hashtag, \#30S cumplió su función de unificar la búsqueda del hecho, señalar el día y ser un código de comunicación dentro de Internet $^{10}$.

FIGURA 1
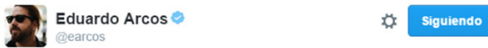

propongo \#30S como hashtag corto para identificar lo que pasa en \#ecuador

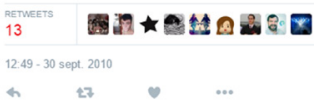

Fuente: Captura obtenida de la cuenta de @earcos y que se muestra en noticia de El Comercio en su formato original de $2010^{11}$

\footnotetext{
${ }^{8}$ De acuerdo con Cristian Espinosa (2010) "Twitter creció en forma casi vertical en el 2010, cuando en el 2009 Google Trends ni siquiera registraba visitas de Twitter en Ecuador"

${ }^{9}$ Se usa la hashtag \#30S en el presente trabajo para fines meramente académicos.

${ }^{10}$ El hashtag es el símbolo \# para marcar palabras y temas clave de un tuit.

${ }^{11}$ Véase nota: El Comercio (2011, 13 de diciembre). Bloguero Eduardo Arcos se opone al registro de 30-S como marca. Política. Recuperado de http:// www.elcomercio.com/actualidad/politica/bloguero-eduardo-arcos-se-opone.html
}

La situación del tema cambió en el transcurso del año siguiente al motín policial. En este período el gobierno tomó cartas en la mediación del hecho, por lo cual los sucesos del 30 de septiembre se convirtieron en un importante momento de la memoria histórica, recordado varias veces en el discurso oficial como un día en que la democracia se adelantó a los intereses golpistas. Por ejemplo, al año del acontecimiento se realizaron misas católicas por las 10 personas fallecidas, programas especiales y convocatorias (periodistasenespañol.org, 2011). Igualmente existe http:// www.el30s.com/ una página conmemorativa que muestra documentación sobre el hecho.

Adicionalmente, en 2012 el Estado ecuatoriano medió el uso de la mencionada hashtag \#30S, pues se procedió a su registro y al de frases similares en el Instituto Ecuatoriano de Propiedad Intelectual $-\mid \mathrm{EPI}^{12}{ }^{12}$, restringiendo su uso a ciertas actividades que se señalarán en

12 “. ..19 de mayo de 1998 se crea un solo organismo con el fin de agrupar todas las áreas de la protección a la creación y es cuando se publica en el Registro Oficial No. 320, en la nueva Ley de Propiedad Intelectual, la instauración del Instituto Ecuatoriano de la Propiedad Intelectual IEPI que"... ejercerá las atribuciones y competencias establecidas por la Ley de Propiedad Intelectual... será considerado como la oficina nacional competente para los efectos previstos en las decisiones de la Comisión de la Comunidad Andina... Recuperado de http://www. iepi.gob.ec/index.php/quienes-somos 
el apartado de análisis. Así, en el presente trabajo se tomará este caso como un ejemplo de la segurización desde el Estado, una acción de la seguridad nacional que no solo se juega en el espacio público, sino también en el virtual, puesto que una de las frases registradas nació y se propagó primero en la Red.

En el siguiente apartado se hace un relato más profundo del caso en los medios de comunicación ecuatorianos. Para ello se usarán tres periódicos: a) el medio público El Telégrafo, b) el portal digital Ecuadorinmediato.com y el c) el rotativo privado El Comercio. Se aplicarán en los textos el análisis del discurso, el modelo de la segurización de Buzan (Buzan, et.al., 1998) y los lineamientos foucaultianos de la biopolítica. Para esto, se hará antes una profundización teórica.

\section{Algunas anotaciones teóricas}

Las acciones seguritarias en redes sociales que no entran en el plano económico, militar o ambiental, ingresan conceptualmente en lo que se determina seguridad política y seguridad societal (Buzan, et. al., 1998). La primera se refiere a contribuir a la estabilidad de los Estados y de los sistemas de gobierno, así como al control de ideologías que podrían atentar en contra de los intereses estatales. La seguridad que va a lo social apunta más bien a la sostenibilidad de los modelos de lenguaje, religión, cultura, identidad nacional y costumbres (Buzan, et. al., 1998). No hay que olvidar que la Doctrina de Seguridad Nacional, muy difundida en América Latina, concibe a la cultura y "moral" de la sociedad como uno de los recursos que deben presentarse para mantener y proteger a la nación ${ }^{13}$.

Debido a la necesidad que tiene el Estado de protección a su espacio y a su estructura, se procede en dos planos o niveles: el primero se refiere a un proceso de "politización" -introducción de un asunto en la agenda de política pública-. En cambio, si se habla ya de una amenaza, se procede a una acción segurizadora la cual indica un peligro existencial que lleva a medidas de emergencia y/o medidas al borde de lo legal (Buzan, et.al., 1998). Estas amenazas pueden ser de todo tipo, no solo de poder duro, sino también políticas y sociales.

En este sentido, la comunicación en red es un elemento de acción social y política, que puede transformarse en una amenaza para las instituciones estatales y la seguridad nacional. De hecho, en las acciones de ciberseguridad ya no

${ }^{13}$ La Doctrina, que primó en la Guerra Fría, priorizaba la seguridad del Estado en base al control de la sociedad, objetivo logrado a través del control militar de la institucionalidad estatal. Se sustituye la figura del enemigo externo por un enemigo interno, el cual se determinaba en agentes locales supuestamente trabajando para el comunismo. ..."(Buitrago, 2003). 
ReVista PUCE, ISSN 1390-7719. Núm.103. 3 de mAYO de 2016-

3 de noviembre de 2016. Fredy Rivera Vélez, Gilda Alicia

GUERRERO SALGADO. PP. 233-252

se habla simplemente de ataques físicos desde la red hacia lo público- la suplantación de identidades, el "phishing"14, la trata de personas a través de páginas web y redes sociales, entre otros -, sino que se habla del poder de la información como un peligro. Así, el ciberespacio es otro campo de batalla: habría una delgada línea que separaría activismo y delincuencia (Caro Bejarano, 2010).

De hecho, el ciberespacio, palabra acuñada por William Gibson en la novela "Neuromancer"15, vive ya la apropiación desde el Estado. Aunque en rigor lo "ciber" e Internet son una creación colectiva a nivel mundial, los Estados buscan enlazar lo virtual con el espacio estatal, fuera de sus elementos clásicos: gobierno, soberanía, territorio y población.

Por ejemplo, existen casos como la mencionada red White House2 en Estados Unidos, mientras que en el mismo país se intentó introducir en el Poder Legislativo la Ley SOPA. Esta ley, conocida como Stop Online Piracy Act, tuvo una propuesta gemela PIPA: Protect Intelectual Property Act. Estas fueron iniciativas que en 2011 estuvieron pendientes de aprobación por el Congreso y el Senado

\footnotetext{
${ }^{14}$ Robo o fraude financiero a través de la red.

15 “...Una representación gráfica de los datos abstraídos por los bancos en cada computadora en el sistema humano. Complejidad inimaginable. Líneas e luz que se desplazan en el no espacio de la mente, clusters y constelaciones de datos..." (Gibson, 1984: 67, citado en Bell, 2007).
}

estadounidenses con el fin de terminar con la piratería y el robo de material protegido. Incluso, se propuso con estas medidas la criminalización de cualquier uso de material en web con copyright sea este para su venta o no (Pereda, 2012).

Así, no hay gobernanza o normas aceptadas. Parecería que el manejo de la Red Mundial se encuentra dentro de la anarquía en que vive el Sistema Internacional: cada unidad decide de acuerdo con su interés "(self help)", mientras que no hay una jerarquía externa o Derecho para que los Estados determinen algún tipo de acción vinculante (Donelly, 2000).

Con estos antecedentes, se observa que la comunicación en Web es un elemento social y político que puede mediarse por el gobierno y que puede ser segurizado. También, se ha visto cómo el Estado es capaz de controlar a los intercambios en línea de manera biológica; es decir, mesura, media y diagnostica para la integridad del cuerpo de la nación. De esta manera, la segurización es una forma de trabajo del biopoder, una biopolítica. Como indicó Foucault, con estos procesos se aspira a la homeostasis: la seguridad del conjunto por medio de los procesos internos (Foucault, 2006: 15-22). Desde este enfoque teórico se realizará el análisis de caso en los siguientes apartados. 


\section{Discusión: un ciberespacio segurizado}

En 2011, un año después de los acontecimientos de ese 30 de septiembre de 2010, se introdujo una solicitud del gobierno ecuatoriano en el Instituto Ecuatoriano de Propiedad Intelectual, IEPI. La solicitud pedía el registro como propiedad del Estado de las frases "30S", “30-S" y "Prohibido Olvidar". Con esta estrategia el aparato estatal estaría en la capacidad de exigir regalías, cobrar licencias y solicitar acciones legales por uso indebido de las expresiones (Ecuador Inmediato, 2012, 30 de agosto). A esto, según señala el IEPI, se añadiría una expresión más: “30S el día que triunfó la democracia". Todas las solicitudes, al ser de registro normales, pasaron el procedimiento establecido y por cada uno de los exámenes técnicos. (IEPI, 2012) ${ }^{16}$.

La Secretaría Nacional de la Administración Pública, señaló la normalidad del proceso con el fin de "precautelar los iconos que podrían ser considerados o convertirse en elementos con valor patrimonial, histórico o promocional en beneficio del país." (El Telégrafo, 2011, 16 de diciembre) y con el fin de "evitar usos inapropiados o en desmedro de la imagen del país o de los

\footnotetext{
${ }^{16}$ Véase el comunicado oficial de IEPI (2012) en noticias del Ministerio Coordinador de Conocimiento y Talento Humano. Recuperado de http://www. conocimiento.gob.ec/el-registro-de-marcas-tiene-multiples-ventajas/
}

hechos, circunstancias o acontecimientos que los motivaron." (El Telégrafo, 2011, 16 de diciembre) ${ }^{17}$. De hecho, el IEPI en su comunicado sobre la solicitud del registro señaló que existían ejemplos a nivel internacional con el registro de marcas como "SEPTEMBER 11"; "NATIONAL SEPTEMBER 11 MEMORIAL MUSEUM" entre otras, que fueron registradas en The United States Patent and Trademark Office de los Estados Unidos, con el fin de conmemorar los hechos que sucedieron el 11 de septiembre de 2001 en Nueva York. Se señaló, también, que este tipo de acciones impulsarían el registro de marcas en el Ecuador. (IEPI, 2012)

El proceso tardó alrededor de un año para "evitar el posible mal uso y distorsión con fines políticos o de otra índole, dado que el $\mathbf{3 0}$ de septiembre ocurrió un suceso de connotación nacional e internacional que llevó a la ciudadanía a salir a las calles a defender la democracia y condenar el intento de golpe de Estado". (E) Telégrafo, 2011, 16 de diciembre, negritas de los autores).

Adicionalmente, se hizo la aclaración que, a pesar del registro, las frases pueden usarse "para fines informativos o identificativos" siempre que estos estén bajo los lineamientos de la buena fe, no se persiga fines comerciales y no se haga

${ }^{17}$ Se cita en este medio a otro portal público, llamado El Ciudadano. Los periódicos aquí citados son de ediciones digitales sin paginación. 
ReVista PUCE, ISSN 1390-7719. Núm.103, 3 de mAYO de 2016-

3 de noviembre de 2016. Fredy Rivera Vélez, Gilda Alicia

a título de marca (El Telégrafo, 2011, 16 de diciembre). Todo este quehacer legal se realizó bajo la Ley de Propiedad Intelectual [de Ecuador] y la decisión 486 de la Comunidad Andina de Naciones ${ }^{18}$, una decisión a la que podía oponerse, de acuerdo a la Ley, cualquier persona que tenga un legítimo interés (El Telégrafo, 2011, 16 de diciembre). El proceso terminó finalmente con la aceptación del registro de las frases desde el 26 de julio de 2012 hasta mayo de 2022 en la Gaceta 560 (Ecuador Inmediato, 2012, 30 de agosto).

Se observa entonces un proceso legal para la posesión de una frase dentro del interés nacional, que se entiende como preservación y estabilidad del Estado. ¿Por qué se dice entonces que esta "hashtag" fue segurizada? ¿Por qué esta segurización está ligada a un uso del biopoder en la política?

\section{Resultados: una "hashtag" en la biopolítica}

Al retomar la hipótesis en el caso ecuatoriano, se puede enunciar que, en el registro de las frases relativas al 30 de septiembre de 2010 como propiedad intelectual del Estado ecuatoriano, el proceso fue de apropiación con el fin de

18 Se refiere al Régimen Común sobre Propiedad Intelectual de los países pertenecientes a la Comunidad Andina de Naciones (Lima, 14 de septiembre de 2000). Véase el documento completo en: http:// www.dib.unal.edu.co/cip/pi_can_decision486.pdf proteger la integridad de la estructura estatal ecuatoriana, un proceso incipiente pero determinante de segurización de un elemento que se crea en redes sociales que en los términos del biopoder, sirvió también para la mantener la "buena salud" del Estado ecuatoriano.

Si se examinan los significantes "precautelar íconos", "mal uso", "distorsión" y "fines políticos" sobre el registro de las frases (El Telégrafo, 2011, 16 de diciembre), se ve que la estructura gubernamental ecuatoriana buscó mantener la identidad, la institucionalidad y la memoria histórica del hecho. Evitar en todo sentido una acción no consentida, fuera de las instancias estatales.

Esta acción fue segurizadora porque se determinó desde el poder para la cohesión estatal y el cuidado y protección de la sociedad asumidas como conjunto. De hecho, el teórico realista de las Relaciones Internacionales, Kenneth Waltz advierte que el sistema internacional es una estructura con unidades estatales que tienen diferente riqueza, poder, tamaño y que luchan por su supervivencia en un estado de Guerra continuo (Waltz, 1988: 144). Con esto en mente, se puede entender que la variable poder, que es la microestructura no explicitada, pero presente en las noticias citadas sobre el registro del \#30S, es una de las grandes metas del Estado, el cual, con ese poder, une a los ciudadanos que 
aceptan las reglas con el fin de mantenerse en comunidad y en seguridad.

De hecho, la Ley de Seguridad Pública y del Estado (Ecuador, 2009) señala el amparo integral de los ecuatorianos en aspectos que van desde la soberanía territorial hasta las instituciones, pasando por las personas, comunidades, pueblos, nacionalidades, y colectivos. Adicionalmente, "en el ámbito de la seguridad del Estado la protección y control de los riesgos tecnológicos y científicos, la tecnología e industria militar, el material bélico, tenencia y porte de armas, materiales, sustancias biológicas y radioactivas, etc." (Artículo 2, Ley de Seguridad Pública de Ecuador, 2009). Es decir, la garantía de la seguridad desde el Estado ecuatoriano abarca todos los contextos tecnológicos, públicos y privados $^{19}$. Se da la acción

19 "Art. 2.- De los ámbitos de la ley.- Al amparo de esta ley se establecerán e implementarán políticas, planes, estrategias y acciones oportunas para garantizar la soberanía e integridad territorial, la seguridad de las personas, comunidades, pueblos, nacionalidades y colectivos, e instituciones, la convivencia ciudadana de una manera integral, multidimensional, permanente, la complementariedad entre lo público y lo privado, la iniciativa y aporte ciudadanos, y se establecerán estrategias de prevención para tiempos de crisis o grave conmoción social.

Se protegerá el patrimonio cultural, la diversidad biológica, los recursos genéticos, los recursos naturales, la calidad de vida ciudadana, la soberanía alimentaria; y en el ámbito de la seguridad del Estado la protección y control de los riesgos tecnológicos y científicos, la tecnología e industria militar, el material bélico, tenencia y porte de armas, materiales, sustancias biológicas y radioactivas, etc." (Ley de Seguridad Pública y del Estado, 2009). porque el Estado tiene poder sobre todo ámbito, sobre toda su corporalidad, incluida la parte virtual.

En cambio, en la ciudadanía hubo cuestionamientos. Eduardo Arcos, el creador de la "hashtag \#30S", indicó que "Es un término público, el cual ha sido utilizado por los ciudadanos sin restricción alguna, convirtiéndose en un genérico para referirse a una fecha" (El Comercio, 2011, 14 de diciembre). El registro sería una"restricción de su uso para referirse a un hecho (...) en construcción", por lo que "nuevas piezas literarias, gráficas o videográficas, entre otras, que aporten información no podrían usar de manera libre el nombre genérico". (El Comercio, 2011, 14 de diciembre).

Otro experto citado en la misma nota (Juan Carlos Donoso), señaló con el temor que el gobierno ecuatoriano acaparara el debate y señaló que la patente "evitará que eventualmente nazca algún movimiento con los nombres 30-So Prohibido olvidar..." (El Comercio, 2011, 14 de diciembre). Es decir, se observó una inquietud de la ciudadanía frente al hecho con el significante "restricción". Hubo dudas sobre los usos legales y las implicaciones de la acción emergente.

Adicionalmente, en lo "no dicho" del discurso -es decir, las microestructuras o lazos de poder-, se encuentra la categoría de "Io público" que se refiere al acceso ciudadano. El Estado medió lo público para transformar un elemento 
ReVista PUCE, ISSN 1390-7719. Núm.103. 3 de mAYO de 2016-

3 de noviembre de 2016. Fredy Rivera Vélez, Gilda Alicia

GUERRERO SALGADO. PP. 233-252

de este ámbito en su propiedad desde su instancia de poder, incluso frente a las críticas. Todo con el objetivo de la seguridad.

Igualmente, ligada a la segurización, está la visión biopolítica sobre los acontecimientos relatados. Como se introdujo anteriormente, se dice que el biopoder es la aparición de hechos biológicos y naturalistas en la vida cotidiana $y$, por ende, en todos los aspectos de la vida social: "mecanismos por medio de los cuales aquello que, en la especie humana, constituye sus rasgos biológicos fundamentales podrá ser parte de una política, una estrategia política, una estrategia general de poder" (Lazzarato, 2000). La biopolítica "mueve" al biopoder. De esta manera, en una extensión del pensamiento primigenio foucaultiano, la primera es una herramienta del segundo, pues"la biopolítica se "incorpora"y se "afianza" sobre una multiplicidad de relaciones de mando y de obediencia entre fuerzas que el poder "coordina, institucionaliza, estratifica y concluye", algo que no se refiere a la proyección pura y simple sobre los individuos." (Lazzarato, 2000)20.

Con este análisis, este trabajo propone finalmente que la segurización del hashtag creada en las redes sociales ecuatorianas, durante el 30 de septiembre de 2010 en Ecuador, generó una

\footnotetext{
${ }^{20}$ Edición digital sin paginación.
}

reacción constructivista y biopolítica de protección del Estado, puesto que la etiqueta de Twitter nació de un hecho grave para la seguridad pública y que desestabilizó al Ecuador. Todo esto se hizo para mantener el espacio público de la estructura estatal y para apropiarse y proteger un "Estado virtual".

Ese cuerpo físico del Estado toma un miembro más, -lo virtual- y de ahí elimina lo que se consideraría nocivo. Fue una operación legal-quirúrgica, por la cual se sacaron del dominio público unas frases para la discusión, uso y abuso, con el fin de evitar malos manejos y la inadecuada utilización de símbolos discursivos para cuidar la imagen del Estado. Al mismo tiempo se previenen prácticas que podrían ir en contra de la seguridad pública, de la democracia y de la participación ciudadana. No en vano, se señaló, desde instancias gubernamentales, que el registro evitaría malos usos y distorsiones ante hechos de legitimidad y valor histórico patrimonial, como se indicó más arriba (El Telégrafo, 2011, 16 de diciembre).

El gobierno precautela la vida y las formas de vida de sus gobernados, esto en Ecuador y a nivel internacional. El Estado toma como ámbito de su quehacer de seguridad al espacio virtual y al espacio público de los ciudadanos. Los moldea de acuerdo con sus necesidades que son desde la biopolítica, las necesi- 
dades de todos, porque el poder político logra tocar los cuerpos, aferrarse a ellos y disciplinarlos (Foucault, 2005). El Estado retira o pone en cuarentena un fenómeno a través de la segurización para mantener la salud de la paz social.

El biopoder del Estado se une a la segurización al tomar un hecho públi- co nacional/virtual y al transformarlo en una biopolítica. Es decir, desde lo legal se retira de la circulación el posible "virus" del mal uso de las frases registradas que podían afectar al Estado y la protección que despliega hacia la sociedad como una garantía.

\section{CONCLUSIONES}

En este texto se demuestra que el caso del registro de marca de frases relativas a la insurrección policial en Ecuador del 30 de septiembre de 2010, una acción gestada por el gobierno ecuatoriano, es también una acción de biopoder aplicada para mantener la memoria histórica y la seguridad del Estado nacional. Este proceso tiene su nacimiento en las redes sociales, donde una de las frases (hashtag \#30S) nació y se reprodujo en las discusiones de la red Twitter durante el día de los violentos acontecimientos.

Se prueba entonces que las redes sociales en Internet son un sujeto de observación desde el Estado para su integridad, incluso si están en el ciberespacio. El registro de marca habla de un novedoso proceso de regularización, mediación y segurización, pues el hecho apela al control de una expresión nacida en lo virtual para mantener la institucionalidad.
De esta forma, es posible indicar cómo se vincula la biopolítica con las redes sociales, en tanto que constituyen una dimensión no física y subjetiva. Internet es una construcción conjunta (o cuerpo de la conciencia e ideas) de diferentes seres humanos y, por lo tanto, sujeta al biopoder. En este caso, se observó la manera de operar en las redes por parte del Estado para preservarse a sí mismo, a su memoria y a su identidad. Mientras tanto, la acción colectiva y la discusión sobre el motín se disciplinaron con la segurización, pues con la "cuarentena" que fue el registro del hashtag, estas fueron aisladas y restringidas para que los organismos estatales puedan sobrevivir

La seguridad nacional es una práctica, una garantía y una capacidad legal del poder estatal. Dentro del Sistema Internacional se puede observar cómo hay Estados capaces de mantener 
ReVista PUCE, ISSN 1390-7719. Núm.103. 3 de mAYO de 2016-

3 de noviembre de 2016. Fredy Rivera Vélez, Gilda Alicia

su balance de poder y su estatus a toda costa. Los ciudadanos a través de las redes sociales comienzan a tener un poder individual de expresión y de reproducción de su conocimiento como nunca antes. En ese sentido, el ciberciudadano actúa en la red por medio de estrategias de intercambio, cooperación y prevención para extender contenidos políticos; de hecho, opera bajo el supuesto que existe una democracia interconectada que pone en crisis el concepto tradicional de soberanía ligado al de seguridad nacional.

Con el uso de Twitter, en el contexto de los hechos del 30 de septiembre de 2010, se ve cómo el Estado ecuatoriano se protegió a través de la herramienta legal del registro de la "hashtag \#30S" y convirtió a esta herramienta en un mecanismo de seguridad en contra de posibles amenazas ideológicas, políticas o sociales. Esto se pudo rastrear con significantes visibles en el discurso oficial en medios.

En ese aislamiento de una amenaza (el acrónimo \#30S) el Estado no solo se "vacuna" ante un uso político del hecho que podría ser nocivo, sino que también determina una mayor atención de la Seguridad Nacional hacia las redes sociales, tendencia que parece ser la tónica frente al fenómeno comunicacional de la Web 2.0.

\section{BIBLIOGRAFÍA}

Bell, D. (2007). Cyberculture Theorists.Manuel Castells and Donna Haraway. Inglaterra: Routledge

Buzan, B., WæVER, O. \& DE WILDE, J. (1998). Security a New Framework for Analytical Survey. Boulder: Lynne Rienner Publishers

Caro Bejarano, M.J. (2010). Alcance y ámbito de la seguridad nacional en el ciberespacio. Ciberseguridad. Retos y amenazas a la seguridad nacional en el ciberespacio. Cuadernos de Estrategia. España: Ministerio de Defensa, 149. Diciembre. Recuperado de: http://www. cni.es/comun/recursos/descargas/ Cuaderno_IEEE_149_Ciberseguridad.pdf

Castells, M. (2002). La era de la información. Buenos Aires: Siglo XXI.

De la Torre, C. (2011). Corporatism, Charisma and Chaos. Ecuador's Police Rebellion in Context. Nacla reports on the Americas, Enero/ Febrero, 25-32

Donelly, J. (2000). Realism and International Relations. Cambridge: Cambridge University Press.

Ecuadorinmediato.com (2012, 30 de agosto). Marca 30-S pertenece exclusivamente a Gobierno. Política Nacional. Ecuadorinmediato.com. Recuperado de: http://www.ecuadorinmediato. $\mathrm{com} /$ index.php? module $=$ Noti - 
cias\&func=news_user_view\&i$d=180491 \& u m t=$ marca_30s_ pertenece_exclusivamente_a_ gobierno

El Comercio (2011, 14 de diciembre). El registro de la marca $30-5$ es impugnado. Política. Recuperado de: http://www.elcomercio.com/ politica/registro-marca-impugnado_0_608339374.html

El Telégrafo (2011,16 de diciembre). La terminología del 305 adquiere valor patrimonial. Recuperado de: http://www.telegrafo.com. ec/actualidad/item/la-terminologia-del-30-s-adquiere-valor-patrimonial.html

Coronel, Gabriela (2011). "Redes sociales marcan tendencias en la comunicación: Ecuador y el \#30s". Razón y Palabra. Recuperado de: http:// www.razonypalabra.org.mx/varia/77\%205a\%20parte/73_Coronel_V77.pdf

Crónica (2016, 18 de mayo). Nueve policías juzgados por paralizar servicios públicos el 30S. Crónica. Recuperado de http://cronica.com. ec/informacion/item/12904-nueve-policias-juzgados-por-paralizar-servicios-publicos-el-30s

Foucault, M. (2005). El poder Psiquiátrico: Curso en el Collège de France (19751976). Argentina: Fondo de Cultura Económica.
Foucault, M. (2006). Seguridad, Territorio y Población. Curso en el Collège de France (1977-1978). Buenos Aires: Fondo de Cultura Económica.

Griffiths, J. E. (2007). Seguridad Hemisférica en América Latina. Alcances y Proposiciones. Revista Globalización, Competitividad, y Gobernabilidad. Estados Unidos: Universidad de Georgetown. 1(1). Recuperado de: http://www. resdal.org/ultimos-documentos/ articulo-john-griffiths.pdf

Espinosa, C. (2010, 28 de octubre) Twitter Ecuador:2010,elañodel crecimiento. Cobertura digital. Recuperado de: http://www.coberturadigital. com/2010 /10/28/twitter-ecuador-2010-el-ano-del-crecimiento/ Lazzarato, M. (2000). "Del Biopoder a la Biopolítica". Revista Multitudes, 1 (marzo). Francia. Recuperado de: http://www.sindominio.net/arkitzean/otrascosas/ lazzarato.htm.

Levy, P. (2011). Cibercultura. La cultura de la sociedad digital. Anthropos: Barcelona.

Maldonado, C. \& Urán, O. (2007). La configuración del espacio tiempo social y las redes de acción colectiva en Internet. Católica del Norte: Fundación Universitaria, 22, 1-23-

Ministerio Coordinador de Conocimiento y Talento Humano (2012). El registro de marcas tiene múltiples 
ReVista PUCE, ISSN 1390-7719. Núm.103. 3 de mAYO de 2016-

3 de noviembre de 2016. Fredy Rivera Vélez, Gilda Alicia

ventajas, Comunicado de IEPI. Noticias. Recuperado de http:// www.conocimiento.gob.ec/el-registro-de-marcas-tiene-multiples-ventajas/

Morueco Romera, M. (S/F). El ciberespacio como nuevo espacio político: notas para una ontología política nómada. Ponencia congreso UIB. No especifica fecha. Recuperado de: http://www.uibcongres.org/ imgdb/archivo_dpo1785.pdf

Moreno, M. (2016, 21 de mayo). Facebook ya tiene 1590 millones de usuarios. Trecebits redes sociales y periodismo 2.0. Recuperado de: http:// www.trecebits.com/2016/01/28/ facebook-ya-tiene-1-590-millones-de-usuarios/

Navarro, E. \& García, A. (2011). Nuevos textos y contextos en la web 2.0 estudios de caso relacionados con las revoluciones en el norte de África y Oriente Próximo. CIC: Cuadernos de información y comunicación, 16, 149-195.

Ortiz, S. (2011). 30-S: La vulnerabilidad del liderazgo de la Revolución Ciudadana y de la institucionalidad en Ecuador. Íconos, 39(enero) Quito: FLACSO Sede Ecuador, 25-35

O’Reilly, T. (2006). Qué es Web 2.0. Patrones del diseño y modelos del negocio para la siguiente generación del software. La Sociedad de
Información. España: Fundación Telefónica. 23 de febrero.

Pereda, C. (2012, 19 de enero). Las claves de las leyes SOPA y PIPA. El País. Sección Tecnología. Recuperado de: http://tecnologia.elpais.com/ tecnologia/2012/01/19 /actualidad/1326967261_850215.html

Puyosa, I. (2012). Conectados versus mediáticos ¿politizados o despolitizados? Anuario electrónico de estudios en Comunicación Social. Universidad Central de Venezuela. (5)1, 160-183. Recuperado de: http://dialnet.unirioja.es/servlet/ articulo?codigo $=4041942$

Periodistas en español.org (2011, 30 de septiembre). Ecuador: se cumple un año del golpe de Estado del 305 contra Rafael Correa. Política. Periodistas en español. org. Recuperado de: http://www. periodistas-es.org/politica/ecuador-se-cumple-un-ano-del-golpe-de-estado-del-30s-contra-rafael-correa

República del Ecuador (2009). Ley de seguridad pública y del Estado. Ecuador: Registro Oficial No. 35. 28 de septiembre.

Rivera, F. (2012). La Seguridad Perversa. Quito: FLACSO Ecuador

Rivera, F. \& Guerrero, G. (2014). Web 2.0 and National Security Strategies: Similarities Between and Perspec- 
tives on Spain and Ecuador. The Cases of \#15M and \#30S. Aijss. Recuperado de: http://www.aijssnet.com/journals/ Vol_3_No_2_ March_2014/20.pdf

Román, C. \& Urán, O. (2007). La configuración del espacio-tiempo social y las redes de acción colectiva en Internet. Marzo. Católica del Norte. Fundación Universitaria.

Sánchez, L. (2011). Elementos para el análisis del discurso en político en el escenario internacional. Revista Internacional de Pensamiento Político. CERPI, IRI, Argentina: Universidad Nacional de La Plata, 6, 419-433.

Savanoni, R. (2010). Democracia, innovación y cultura digital. Digithum. España: Universidad Abierta de Cataluña.

Van Dijk, T. (1997). Racismo y análisis crítico de los medios. España: Paidós.

Watts, J. (2006, 20 de febrero). War of the Words. The Guardian. Media \& News Features. Recuperado de: http://www.guardian.co.uk/technology/2006/feb/20/news.mondaymediasection

Waltz, K. (1988). Teoría de la Política Internacional. Buenos Aires: GEL Editor 